\title{
THE STABILITY OF COSMOLOGICAL SCALING SOLUTIONS
}

\author{
A. P. Billyard ${ }^{1}$, A. A. Coley ${ }^{1}$ And R. J. van den Hoogen ${ }^{2}$ \\ ${ }^{1}$ Department of Mathematics, \\ Statistics and Computing Science \\ Dalhousie University \\ Halifax, Nova Scotia B3H 3J5 \\ ${ }^{2}$ Department of Mathematics, \\ Statistics, and Computer Science \\ Saint Francis Xavier University \\ Antigonish, Nova Scotia B2G 2W5
}

\begin{abstract}
.
We study the stability of cosmological scaling solutions within the class of spatially homogeneous cosmological models with a perfect fluid subject to the equation of state $p_{\gamma}=(\gamma-1) \rho_{\gamma}$ (where $\gamma$ is a constant satisfying $0<\gamma<2$ ) and a scalar field with an exponential potential. The scaling solutions, which are spatially flat isotropic models in which the scalar field energy density tracks that of the perfect fluid, are of physical interest. For example, in these models a significant fraction of the current energy density of the Universe may be contained in the scalar field whose dynamical effects mimic cold dark matter. It is known that the scaling solutions are late-time attractors (i.e., stable) in the subclass of flat isotropic models. We find that the scaling solutions are stable (to shear and curvature perturbations) in generic anisotropic Bianchi models when $\gamma<2 / 3$. However, when $\gamma>2 / 3$, and particularly for realistic matter with $\gamma \geq 1$, the scaling solutions are unstable; essentially they are unstable to curvature perturbations, although they are stable to shear perturbations. We briefly discuss the physical consequences of these results.
\end{abstract}

\section{Introduction.}

Scalar field cosmological models are of great importance in the study of the early Universe. Models with a variety of self-interaction potentials have been studied, and one potential that is commonly investigated and which arises in a number of 
physical situations has an exponential dependence on the scalar field $[1-5]$. There have been a number of studies of spatially homogeneous scalar field cosmological models with an exponential potential, with particular emphasis on the possible existence of inflation in such models [1].

These models may also be important even if the exponential potential is too steep to drive inflation. For example, there exist 'scaling solutions' in which the scalar field energy density tracks that of the perfect fluid (so that at late times neither field is neglible) [2]. In particular, in [3] a phase-plane analysis of the spatially flat Friedmann-Robertson-Walker (FRW) models showed that these scaling solutions are the unique late-time attractors whenever they exist. The cosmological consequences of these scaling models have been further studied in [4]. For example, in such models a significant fraction of the current energy density of the Universe may be contained in the homogeneous scalar field whose dynamical effects mimic cold dark matter; the tightest constraint on these cosmological models comes from primordial nucleosynthesis bounds on any such relic density [2-4].

Clearly these scaling models are of potential cosmological significance. It is consequently of prime importance to determine the genericity of such models by studying their stability in the context of more general spatially homogeneous models. It is this question that we shall address in this paper.

\section{The Scaling Solution.}

The governing equations for a scalar field with an exponential potential

$$
V=V_{0} e^{-\kappa \phi}
$$

where $V_{0}$ and $\kappa$ are positive constants, evolving in a flat FRW model containing a separately conserved perfect which satisfies the barotropic equation of state

$$
p_{\gamma}=(\gamma-1) \rho_{\gamma}
$$

where the constant $\gamma$ satisfies $0 \leq \gamma \leq 2$ (although we shall only be interested in the range $0<\gamma<2$ here), are given by

$$
\begin{aligned}
\dot{H} & =-\frac{1}{2}\left(\gamma \rho_{\gamma}+\dot{\phi}^{2}\right), \\
\dot{\rho}_{\gamma} & =-3 \gamma H \rho_{\gamma}, \\
\ddot{\phi} & =-3 H \dot{\phi}+\kappa V,
\end{aligned}
$$

subject to the Friedmann constraint

$$
H^{2}=\frac{1}{3}\left(\rho_{\gamma}+\frac{1}{2} \dot{\phi}^{2}+V\right)
$$


where $H$ is the Hubble parameter, an overdot denotes ordinary differentiation with respect to time $t$, and units have been chosen so that $8 \pi G=1$. We note that the total energy density of the scalar field is given by

$$
\rho_{\phi}=\frac{1}{2} \dot{\phi}^{2}+V
$$

Defining

$$
x \equiv \frac{\dot{\phi}}{\sqrt{6} H} \quad, \quad y \equiv \frac{\sqrt{V}}{\sqrt{3} H},
$$

and the new logarithmic time variable $\tau$ by

$$
\frac{d \tau}{d t} \equiv H
$$

equations $(2)-(4)$ can be written as the plane-autonomous system [3]:

$$
\begin{aligned}
& x^{\prime}=-3 x+\sqrt{\frac{3}{2}} \kappa y^{2}+\frac{3}{2} x\left[2 x^{2}+\gamma\left(1-x^{2}-y^{2}\right)\right], \\
& y^{\prime}=\frac{3}{2} y\left[-\sqrt{\frac{2}{3}} \kappa x+2 x^{2}+\gamma\left(1-x^{2}-y^{2}\right)\right],
\end{aligned}
$$

where a prime denotes differentiation with respect to $\tau$, and equation (5) becomes

$$
\Omega+\Omega_{\phi}=1
$$

where

$$
\Omega \equiv \frac{\rho_{\gamma}}{3 H^{2}}, \quad \Omega_{\phi} \equiv \frac{\rho_{\phi}}{3 H^{2}}=x^{2}+y^{2},
$$

which implies that $0 \leq x^{2}+y^{2} \leq 1$ for $\Omega \geq 0$ so that the phase-space is bounded.

A qualitative analysis of this plane-autonomous system is given in [3]. The wellknown power-law inflationary solution for $\kappa^{2}<2$ [1] corresponds to the equilibrium point $x=\kappa / \sqrt{6}, y=\left(1-\kappa^{2} / 6\right)^{1 / 2}\left(\Omega_{\phi}=1, \Omega=0\right)$ of the system $(9) /(10)$, which is shown to be stable (i.e., attracting) for $\kappa^{2}<3 \gamma$ in the presence of a barotropic fluid. Previous analysis has shown that when $\kappa^{2}<2$ this power-law inflationary solution is a global attractor in spatially homogeneous models in the absence of a perfect fluid (except for a subclass of Bianchi type IX models which recollapse).

In addition, for $\gamma>0$ there exists a scaling solution corresponding to the equilibrium point

$$
x=x_{0}=\sqrt{\frac{3}{2}} \frac{\gamma}{\kappa}, \quad y=y_{0}=\left[3(2-\gamma) \gamma / 2 \kappa^{2}\right]^{\frac{1}{2}},
$$


whenever $\kappa^{2}>3 \gamma$. The linearization of system (9)/(10) about the equilibrium point (13) yields the two eigenvalues with negative real parts

$$
-\frac{3}{4}(2-\gamma) \pm \frac{3}{4 \kappa} \sqrt{(2-\gamma)\left[24 \gamma^{2}-\kappa^{2}(9 \gamma-2)\right]}
$$

when $\gamma<2$. The equilibrium point is consequently stable (a spiral for $\kappa^{2}>$ $24 \gamma^{2} /(9 \gamma-2)$, else a node) so that the corresponding cosmological solution is a late-time attractor in the class of flat FRW models in which neither the scalar-field nor the perfect fluid dominates the evolution. The effective equation of state for the scalar field is given by

$$
\gamma_{\phi} \equiv \frac{\left(\rho_{\phi}+p_{\phi}\right)}{\rho_{\phi}}=\frac{2 x_{0}^{2}}{x_{0}^{2}+y_{0}^{2}}=\gamma
$$

which is the same as the equation of state parameter for the perfect fluid. The solution is referred to as a scaling solution since the energy density of the scalar field remains proportional to that of the barotropic perfect fluid according to $\Omega / \Omega_{\phi}=$ $\kappa^{2} / 3 \gamma-1[2]$. Since the scaling solution corresponds to an equilibrium point of the system $(9) /(10)$ we note that it is a self-similar cosmological model [6].

\section{Stability of the Scaling Solution.}

Let us study the stability of the scaling solution with respect to anisotropic and curvature perturbations within the class of spatially homogeneous models.

\section{A. Bianchi I models.}

In order to study the stability of the scaling solution with respect to shear perturbations we shall first investigate the class of anisotropic Bianchi I models, which are the simplest spatially homogeneous generalizations of the flat FRW models which have non-zero shear but zero three-curvature. The governing equations in the Bianchi I models are equations (3) and (4), and equation (5) becomes

$$
H^{2}=\frac{1}{3}\left(\rho_{\gamma}+\frac{1}{2} \dot{\phi}^{2}+V\right)+\Sigma^{2}
$$

where $\Sigma^{2}=\frac{1}{3} \Sigma_{0}^{2} R^{-6}$ is the contribution due to the shear, where $\Sigma_{0}$ is a constant and $R$ is the scale factor. Equation (2) is replaced by the time derivative of equation (15).

Using the definitions (7), (8) and (12) we can deduce the governing ordinary differential equations. Due to the $\Sigma^{2}$ term in (15) we can no longer use this equation to substitute for $\rho_{\gamma}$ in the remaining equations, and we consequently obtain the 
three-dimensional autonomous system:

$$
\begin{aligned}
x^{\prime} & =-3 x+\sqrt{\frac{3}{2}} \kappa y^{2}+\frac{3}{2} x\left[2+(\gamma-2) \Omega-2 y^{2}\right], \\
y^{\prime} & =\frac{3}{2} y\left\{-\sqrt{\frac{2}{3}} \kappa x+2+(\gamma-2) \Omega-2 y^{2}\right\}, \\
\Omega^{\prime} & =3 \Omega\left\{(\gamma-2)(\Omega-1)-2 y^{2}\right\},
\end{aligned}
$$

where equation (15) yields

$$
1-\Omega-x^{2}-y^{2}=\Sigma^{2} H^{-2} \geq 0,
$$

so that we again have a bounded phase-space.

The scaling solution, corresponding to the flat FRW solution, is now represented by the equilibrium point

$$
x=x_{0}, y=y_{0}, \Omega=1-\frac{3 \gamma}{\kappa^{2}} .
$$

The linearization of system (16) - (18) about the equilibrium point (20) yields three eigenvalues, two of which are given by (14) and the third has the value $-3(2-\gamma)$, all with negative real parts when $\gamma<2$. Consequently the scaling solution is stable to Bianchi type I shear perturbations.

\section{B. Curved FRW models.}

In order to study the stability of the scaling solution with respect to curvature perturbations we shall first study the class of FRW models which have curvature but no shear. Again equations (3) and (4) are valid, but in this case equation (5) becomes

$$
H^{2}=\frac{1}{3}\left(\rho_{\gamma}+\frac{1}{2} \dot{\phi}^{2}+V\right)+K,
$$

where $K=-k R^{-2}$ and $k$ is a constant that can be scaled to $0, \pm 1$. Equation (2) is again replaced by the time derivative of equation (21).

As in the previous case we cannot use equation (21) to replace $\rho_{\gamma}$, and using the definitions (7), (8) and (12) we obtain the three-dimensional autonomous system:

$$
\begin{aligned}
x^{\prime} & =-3 x+\sqrt{\frac{3}{2}} \kappa y^{2}+\frac{3}{2} x\left[\left(\gamma-\frac{2}{3}\right) \Omega+\frac{2}{3}\left(1+2 x^{2}-y^{2}\right)\right], \\
y^{\prime} & =\frac{3}{2} y\left\{-\sqrt{\frac{2}{3}} \kappa x+\left(\gamma-\frac{2}{3}\right) \Omega+\frac{2}{3}\left(1+2 x^{2}-y^{2}\right)\right\}, \\
\Omega^{\prime} & =3 \Omega\left\{\left(\gamma-\frac{2}{3}\right)(\Omega-1)+\frac{2}{3}\left(2 x^{2}-y^{2}\right)\right\},
\end{aligned}
$$


where

$$
1-\Omega-x^{2}-y^{2}=K H^{-2} .
$$

The phase-space is bounded for $k=0$ or $k=-1$, but not for $k=+1$.

The scaling solution again corresponds to the equilibrium point (20). The linearization of system $(22)$ - (24) about this equilibrium point yields the two eigenvalues with negative real parts given by $(14)$ and the eigenvalue $(3 \gamma-2)$. Hence the scaling solution is only stable for $\gamma<\frac{2}{3}$. For $\gamma>\frac{2}{3}$ the equilibrium point (20) is a saddle with a two-dimensional stable manifold and a one-dimensional unstable manifold.

Consequently the scaling solution is unstable to curvature perturbations in the case of realistic matter $(\gamma \geq 1)$; i.e., the scaling solution is no longer a late-time attractor in this case. However, the scaling solution does correspond to an equilibrium point of the governing autonomous system of ordinary differential equations and hence there are cosmological models that can spend an arbitrarily long time 'close' to this solution. Moreover, since the curvature of the Universe is presently constrained to be small by cosmological observations, it is possible that the scaling solution could be important in the description of our actual Universe. That is, not enough time has yet elapsed for the curvature instability to have effected an appreciable deviation from the flat FRW model (as in the case of the standard perfect fluid FRW model).

Hence the scaling solution may still be of physical interest. To further study its significance it is important to determine its stability in a general class of spatially homogeneous models. We shall therefore study the stability of the scaling solution in the (general) class of Bianchi type $\mathrm{VII}_{h}$ models, which are perhaps the most physically relevant models since they can be regarded as generalizations of the open (negative-curvature) FRW models.

\section{Bianchi $\mathrm{VII}_{h}$ models.}

The Bianchi $\mathrm{VII}_{h}$ models are sufficiently complicated that a simple coordinate approach (similar to that given above) is not desirable. To study Bianchi $\mathrm{VII}_{h}$ spatially homogeneous models with a minimally coupled scalar field with an exponential potential and a barotropic perfect fluid it is best to employ a group-invariant orthonormal frame approach with expansion-normalized state variables governed by a set of dimensionless evolution equations (constituting a 'reduced' dynamical system) with respect to a dimensionless time subject to a non-linear constraint [6], generalizing previous work in which there is no scalar field [7] and in which there is no matter [8].

The reduced dynamical system is seven-dimensional (subject to a constraint) [9]. The scaling solution is again an equilibrium point of this seven-dimensional system. This equilibrium point, which only exists for $\kappa^{2}>3 \gamma$, has two eigenvalues given 
by (14) which have negative real parts for $\gamma<2$, two eigenvalues (corresponding to the shear modes) proportional to $(\gamma-2)$ which are also negative for $\gamma<2$, and two eigenvalues (essentially corresponding to curvature modes) proportional to

$(3 \gamma-2)$ which are negative for $\gamma<\frac{2}{3}$ and positive for $\gamma>\frac{2}{3}$ [9]. The remaining eigenvalue (which also corresponds to a curvature mode) is equal to $3 \gamma-4$. Hence for $\gamma<\frac{2}{3}\left(\kappa^{2}>3 \gamma\right)$ the scaling solution is again stable. However, for realistic matter $(\gamma \geq 1)$ the corresponding equilibrium point is a saddle with a (lower) fouror five-dimensional stable manifold (depending upon whether $\gamma>4 / 3$ or $\gamma<4 / 3$, respectively).

\section{Discussion.}

Perhaps these stability results can be understood heuristically as follows. From the conservation law the barotropic matter redshifts as $R^{-3 \gamma}$. In subsection III.A we saw that in this case the shear $\Sigma^{2}$ redshifts as $R^{-6}$ and so always redshifts faster than the matter, resulting in the stability of the scaling solution. We note that the bifurcation that occurs at $\gamma=2 / 3$ in subsection III.B corresponds to the case in which the curvature $K$ is formally equivalent to a barotropic fluid with $\gamma=2 / 3$, and in which both the matter and the curvature redshift as $R^{-2}$. For $\gamma>2 / 3$, the barotropic matter redshifts faster than $R^{-2}$ and the curvature eventually dominates. A complete qualitative analysis of cosmological models with a perfect fluid and a scalar field with an exponential potential will be undertaken in future work [cf. 9].

\section{REFERENCES}

[1]. J.J. Halliwell, Phys. Lett. B185, 341 (1987); A.B. Burd and J.D. Barrow, Nucl. Phys. B308, 929 (1988); Y. Kitada and K. Maeda, Class. Quantum Grav. 10, 703, (1993); A.A. Coley, J. Ibañez and R.J. van den Hoogen, J. Math. Phys. 38, 5256 (1997).

[2]. C. Wetterich, Nucl. Phys. B302, 668 (1988); D. Wands, E.J. Copeland and A.R. Liddle, Ann. N.Y. Acad. Sci. 688, 647 (1993).

[3]. E.J. Copeland, A.R. Liddle and D. Wands, Phys. Rev. D57, 4686 (1998).

[4]. P.G. Ferreira and M. Joyce, Phys. Rev. Lett. 79, 4740 (1997) and Phys. Rev. D58, 023503 (1998); C. Wetterich, Astron. Astrophys. 301, 321 (1995).

[5]. E.J. Copeland, A. Lahiri and D. Wands, Phys. Rev D50, 4868 (1994).

[6]. J. Wainwright and G.F.R. Ellis, Dynamical Systems in Cosmology, Cambridge University Press (1997).

[7]. C.G. Hewitt and J. Wainwright, Class. Quantum Grav. 10, 99 (1993).

[8]. R.J. van den Hoogen, A.A. Coley and J. Ibañez, Phys. Rev. D55, 5215, (1997).

[9]. A.P. Billyard, A.A. Coley and R.J. van den Hoogen, in preparation (1998). 